\title{
AN EPIDEMIOLOGICAL RETROSPECTIVE ANALYSIS OF THE MICROBIAL FLORA AMONG INFECTED SURGICAL WOUND CASES IN LOCAL SECONDARY \& TERTIARY CARE HOSPITALS.
}

\section{Dr. Sanchita Nihal}

Dr. S.K Gautam*
Asst Professor, Departmart of Microbiology, Raipur Institute of Medical Sciences, Raipur, CG , India.

Asst Professor, Department of Microbiology, Raipur Institute of Medical Sciences, Raipur, CG, India. *Corresponding Author

ABSTRACT Introduction: According to the CDC, one-to-three cases out of 100 surgeries get infected. There is considerable variation in each class according to the type of surgery being performed. The incidence of infection varies from surgeon to surgeon, from hospital to hospital, from one surgical procedure to another and, most importantly, from one patient to another. The aim of this study was to identify the most common microorganism documented in post-operative wound sepsis in surgery wards of local tertiary hospitals situated in Chhattisgarh.

Methods: This Retrospective \& Analytical study involved Prior Consent from Hospital Authorities / Medical Superintendents of the Randomly selected Private Tertiary care hospitals to see the records of the patients from Medical Records Department ( MRD) The study included 100 randomly selected case sheets / records of the patients suffering from SSIs in the various surgical wards in local tertiary care centres. Patients of both sex, age $>14$ years, who had surgical wound pus discharge, with serous or seropurulent discharge and with signs of sepsis present concurrently were included.

Results : Out of the 100 selected case sheets / records of patient's samples processed, In $91 \%$ of records it was found that it yielded aerobic bacterial growth. Monomicrobial growth was seen in 92 samples while 8 samples showed polymicrobial growth. The mean age of the patients was 45.7 years (range 14 to 79 years) and the peak incidence of SSI was observed in age group $>50$ years $(55 \%)$. Among the 100 records / cases, bacterial isolates, S. aureus $(52 \%)$ and E. coli $(24 . \%)$ were the commonest organisms.

Conclusion: . The information obtained from this study allows a better understanding of the microbial etiology of SSIs in local hospitals which may have epidemiological and therapeutic implications. Using the results of this study, an initiative for establishing improved hospital antimicrobial policy and antimicrobial prescribing guidelines can be undertaken.

\section{KEYWORDS : Surgical Site Infections, Retrospective study , Staphylococcus Aureus , E.coli}

INTRODUCTION

Health care-associated infections (HAIs) lay a substantial impact on the patient's morbidity, mortality, hospitalization time, and health care costs.

It is likely that around more than 2 million patients encounter various HAI related illnesses annually. The resistance of the host, if adequate, prevents him from being infected so that no symptoms or signs may be expressed despite the millions of these microorganisms harboured in the organism.

The resistance of the host to the microorganism can be broken either by an increased virulence of the organism or diminished immune status of the host, which may lead to a successful invasion, establishment and subsequent multiplication of these organisms. When a patient gets infected after surgery and the infection is related to that surgery, (usually at the post-operative wound site) it is referred to as post-operative wound sepsis [1,2]. Staphylococcus aureus is commonest organism, most documented, to infect surgical wounds sites. This is because $5 \%$ of people carry the organism on all the hair-bearing areas and up to $50 \%$ of people carry it in their nostrils [3,4]. It is also a transient commensal on the skin of the hand together with Pseudomonas, Klebsiella and Enterobacter species, which are all found on the hands exposed to moisture, abrasions associated with chronic skin disease and nail bed lesions.

The US Centers for Disease Control (CDC) has defined surgical site infection (SSI) as an infection that occurs after surgery in the part of the body where the surgery took place, within 30 days after surgery, or up to one year after surgery in patients receiving implants.[5]

According to the CDC, one-to-three cases out of 100 surgeries get infected. There is, however, considerable variation in each class according to the type of surgery being performed. The incidence of infection varies from surgeon to surgeon, from hospital to hospital, from one surgical procedure to another and, most importantly, from one patient to another.

There is also a gradual change in the bacterial profile of SSI, over time. Now, multidrug-resistant (MDR) strains of bacteria such as Acinetobacter spp. are emerging as pathogens.

The possibility of colonization with multiple antibioticresistant hospitals bred Staphylococcus aureus is a recognized major risk in patient's surgical procedures [1]. Some factors operating in the post-operative period enhance the likelihood of infection in this period [5]. These could be classed as patient related factors, surgery related factors and hospital related factors which increase the likelihood of acquiring post-operative wound sepsis range from age (neonates and the elderly), immune status for the debilitated, drugs including steroids and cytotoxic drugs and other illnesses like diabetes. Surgery related factors include: the type of surgery (a minor surgery is less predisposed than a major surgery); site of surgery whether clean or not; technique of surgery especially avoiding undue tissue injury as dead tissues are potential foci of infection; decreasing surgery dead space as hematomas could accumulate in them leading to surgical wound infection. Deep general anesthesia diminishes immune status unlike local anesthesia [6]. Additional surgical manipulations like open drainage, prosthesis, inadequate bowel preparations with major rerouting of the large bowel are confounding factors of selfinduced wound infections. The length of stay of surgery is also a recognized factor as surgeries lasting over 2 hours are at increased risk of developing surgical wound site infection.

The aim of this study was to identify the most common microorganism documented in post-operative wound sepsis in surgery wards of local tertiary hospitals situated in Chhattisgarh. 


\section{METHEDOLOGY}

This Retrospective \& Analytical study involved Prior Consent from Hospital Authorities / Medical Superintendents of the Randomly selected Private Tertiary care hospitals to see the records of the patients from Medical Records Department ( MRD) with the disclosure that we will use the data for study purpose only. Medical record numbers were used to generate the data for analysis. The study was conducted within ethical standards \& doesn't involved any direct Intervention to any mentioned subjects nor any physical Examination was performed. Randomization was done using computer tables in selecting data. All Patients data had details of standard clinical examinations, routine biochemical and haematological investigations. The study duration was l year. Information on each patient selected were carefully obtained by an in depth study of the patients' case notes (folder), which contain the house officer's detailed clerking at patient's presentation to clinic, casualty or referral before admission to the surgical wards. The day to day conditions of the patients were studied. Every necessary procedure was carefully observed pre-operatively and post-operatively. The symptoms of post-operative wound sepsis like fever, undue pains at the operative site and signs such as exquisite tenderness, hyperaemic incision edges and the discharge of pus from the stitches and incision site (which are indices of wound sepsis) were carefully recorded. A list of swabs taken was made against the organism isolated . A table of bacteria isolated, frequency and percentage of isolation was made. A table to elucidate the organisms in the clean and contaminated operations was also made. A table of antibiogram of the organisms isolated was also prepared (Table 2).

All the cases of Surgical Site Infections (SSIs) that occurred within one month after surgery, in the surgical units, during a period of one year were included in the study. The study included 100 randomly selected case sheets / records of the patients suffering from SSIs in the various surgical wards (orthopedic, general surgery, ophthalmology, obstetrics and gynecology, otorhinolaryngology) in local tertiary care centres . Patients of both sex, age > 14 years, who had surgical wound pus discharge, with serous or seropurulent discharge and with signs of sepsis present concurrently (warmth, erythema, induration, tenderness, pain, raised local temperature) were included.

\section{Exclusion criteria}

Evidence of sepsis at the operative site prior to surgery or pus seen at laparotomy such as drainage of an appendicular mass or pelvic abscess. Oral, anal and urogenital surgeries below the level of the bladder Files with incomplete records were also excluded. The duration of acquisition of the postoperative wound infection was determined by subtracting the date of appearance of pus at the operation site from the date of surgery, one which if less than 4 days was discarded as having been gotten before surgery. Records were rejected if there was any evidence of sepsis at the operative site prior to surgery or pus seen at laparotomy such as drainage of an appendicular mass or pelvic abscess. Patients admitted to the Paediatric Surgery Unit were also excluded from the study.

It was observed in all the cases that the pus was collected using sterile cotton swabs or was aspirated. The identification of bacteria was done based on microscopy, culture and biochemical properties using the standard laboratory procedures. Antibiotic sensitivity testing of bacterial isolates was done using Stokes' disc diffusion method.

It was found in the records that In patients with features of sepsis, blood samples were taken for culture and sensitivity. Detection of Extended-spectrum beta-lactamase (ESBL), AmpC -lactamases, Metallo -lactamase, Carbapenemase production and inducible resistance were done by conventional methods.

Data was filled in Microsoft Excel \& analysed using a computer software Epi Info version 6.2 (Atlanta, Georgia, USA) and SPSS (SPSS Inc., Chicago, Illinois, USA). version 20. P value of 0.05 and less was considered as statistically significant. Results were presented in simple proportions and means ( \pm SD). Chi-square test was also used. Comparison between the study and the control group was performed using the $t$-test for two independent means. Comparison among the subgroups of the study group was carried out using one-way analysis of variance test, and comparison for nonparametric data was carried out using the Fisher exact test.

\section{RESULTS}

Out of the 100 selected case sheets / records of patient's samples processed, In $91 \%$ of records it was found that it yielded aerobic bacterial growth. Monomicrobial growth was seen in 92 samples while 8 samples showed polymicrobial growth. The mean age of the patients was 45.7 years (range 14 to 79 years) and the peak incidence of SSI was observed in age group $>50$ years $(55 \%)$. Table 1 shows the age wise distribution of various morphotypes in SSIs. Males (73\%) were more commonly affected than females $(27 \%)$.

Table 1. Age wise distribution in SSIs

\begin{tabular}{|l|l|l|l|}
\hline Age group in years & Monomicrobial & Polymicrobial & Total \\
\hline $14-50$ & 40 & 5 & 45 \\
\hline$>50$ & 52 & 3 & 55 \\
\hline Total & 92 & 8 & 100 \\
\hline
\end{tabular}

Among the 100 records / cases, bacterial isolates, S. aureus $(52 \%)$ and $E$. coli $(24 . \%)$ were the commonest organisms. Table 2 depicts the characterization of various bacterial isolates obtained from patients with SSIs. Among all 100 cases Antimicrobial susceptibility testing was carried find out for all isolates. Staphylococcus aureus strains showed a high degree of resistance for ampicillin. Methicillin resistance was seen $12 \%$ S.aureus isolates. Gram negative isolates showed even higher rate of resistance and commonly prescribed agents like gentamicin, cotrimoxazole and ciprofloxacin were found resistant for most of the gram negative isolates. Meropenem \& its associated antibiotics showed good activity against most of the gram negative isolates.

Table No.2 Characterization of various bacterial isolates obtained from patients with surgical site infections.

\begin{tabular}{|l|l|}
\hline Organism & Number of Isolates (\%) \\
\hline Staphylococcus Aureus & 52 \\
MSSA & 40 \\
MRSA & 12 \\
\hline Escherichia coli & 24 \\
\hline Pseudomonas aeruginosa & 6 \\
\hline Citrobacter species & 5 \\
\hline Acinetobacter species & 3 \\
\hline Klebsiella species & 8 \\
\hline Proteus species & 2 \\
\hline
\end{tabular}

\section{DISCUSSION}

Despite the advances in surgical techniques and better understanding of the pathogenesis of wound infection, management of SSIs remains a significant concern for surgeons and physicians in a health care facility. Patients with SSIs face additional exposure to microbial populations circulating in a hospital set up which is always charged with microbial pathogens. The unrestrained and rapidly spreading resistance to the available array of antimicrobials further contributes to the existing problem. Most of the SSIs are hospital acquired and vary from hospital to hospital. The rate of SSIs has been reported to be $2.5 \%$ to $41.9 \%$ [6]. 
Various other studies from India have shown the rate of SSI to vary from $6.1 \%$ to $38.7 \%$ [6] . However in comparison to the Indian hospitals the rate of infection reported from other countries is quite low, for instance in USA it is $2.8 \%$ and in European countries it is reported to be $2-5 \%$ [7]. The lack of attention towards the infection control measures, inappropriate hand hygiene practices and over crowded hospitals can be the major contributory factors for high infection rate in Indian hospitals.

The predominance of male patients was seen in this study and this finding was in contrast to the other studies where a much higher number of female patients have been reported [8,9]. The patients with age $>50$ years had a higher incidence of SSI in comparison to an incidence among the patients who were $\leq 50$ years of age. Advancing age is an important factor for the development of SSIs, as in old age patients there is low healing rate, low immunity, increased catabolic processes and presence of co-morbid illness like diabetes, hypertension, etc [10]. Regarding the duration of the operation a prolonged time was found to be a significant risk factor for SSI and it was observed that as the order and the duration of surgery increased, the rate of infection also increased.

Staphylococcus aureus, gram positive cocci, is a major human pathogen and a predominant cause of SSIs worldwide with a prevalence rate ranging from $4.6 \%$ to $54.4 \%$ [11]. In the present study predominance of $S$. aureus was seen and this finding was consistent with reports from other studies $[6,9]$. Infection with $S$. aureus is most likely associated with endogenous source as it is a member of the skin and nasal flora and also with contamination from environment, surgical instruments or from hands of health care workers [12,13]. S. aureus was the single predominant gram positive bacterial isolate obtained in this study. Special interest in S. aureus SSI is mainly due to its predominant role in hospital associated infection and emergence of methicillin resistant S.aureus (MRSA) strains. In our study methicillin resistance was seen in $12 \%$ of S.aureus isolates. This finding was in tandem with the study conducted by Aggarwal et al., [14], who reported methicillin resistance in $10 \%$ of the isolates, however it is in contrast with the study conducted by Kownhar et al., [15] who reported higher incidence of $21.7 \%$. Still higher incidences of $45 \%$ and $58.2 \%$ of MRSA have been documented by Eagye et al., [16] and Kaye et al., [17] respectively. We found that all the $S$. aureus strains (irrespective of methicillin resistance) were sensitive to vancomycin, teicoplanin and linezolid. This finding can be of relevant clinical use for the formulation of antibiotic policy in hospitals.

Gram negative isolates comprised of $48 \%$ of all the aerobic bacterial isolates. $E$. coli was the commonest gram negative bacteria isolated. Similar observations have been reported by various other authors also [8]. Few studies have reported Paeruginosa as the most frequent isolate in SSI [18] Presence of enteric organisms could be attributed to the patient's normal endogenous microbial fecal flora [6] E. coli invasion of the wound is a clear case of poor hospital hygiene.

Antibiotic susceptibility results revealed that a high degree of resistance was seen for majority of the bacterial isolates. For gram positive bacteria vancomycin, teicoplanin, linezolid and amikacin were found to be the most effective antibiotics. The degree of resistance was even higher among the gram negative bacteria and the commonly used drugs were found to be more resistant with an average resistance range from $50 \%$ to $100 \%$. Meropenem, piperacillin-tazobactam, and amikacin were found to be the most effective antimicrobial agents whereas ampicillin, amoxicillin-clavulanate and cefotaxime were among the most resistant drugs The development and spread of resistant bacterial strains has emerged as a global problem. The appearance of multi drug resistant (MDR) strains over the past decades has been regarded as an inevitable genetic response to the strong selective pressure imposed by antimicrobial chemotherapy which plays a crucial role in evolution of antibiotic resistant bacteria.

All cases in our study received prophylactic antimicrobials prior to the surgery. Current recommendations for antimicrobial prophylaxis to prevent SSI advise that an antimicrobial agent be administered within 60 minutes prior to surgery and discontinued soon afterward [19]. More than $50 \%$ of our patients received preoperative antimicrobials more than six hours before surgery and almost all patients were treated with antimicrobials after surgery. Many of them were even treated until the day of discharge in an attempt to prevent infection while they were hospitalized. The most widely used combination was a third generation cephalosporin and an aminoglycoside. The frequent empirical prescription of some antimicrobials as a treatment and prophylaxis in some hospitals might have contributed for observed high degree of resistance. This situation raises a serious concern and calls for immediate revision of antibiotic policy and antibiotic prescribing guidelines.

The rate of SSI observed in this study was comparable to other similar studies carried out in developing countries including India; however the bacterial isolates detected from our patients showed a high degree of resistance for commonly prescribed antimicrobials in our setup. Although the studies with bigger sample size are sought to make it statistically more relevant.

Inspite of the modern surgical and sterilization techniques and the use of prophylactic antimicrobials, SSIs still continue to pose an important clinical challenge. They impose a substantial burden of disease both on patients as well as healthcare services in terms of pharmacotherapeutic and pharmacoeconomic losses. Although SSIs cannot be completely eliminated, but reduction of the rate of infection to minimal can have significant benefits by reducing the wastage of healthcare resources, patient morbidity and mortality. This can be achieved by optimal preoperative, intraoperative and postoperative patient care. Also there is good evidence that attention to multiple patient related and procedure related risk factors can decrease the risk of SSIs significantly. This would be supplemented with proper infection control measures and a sound antibiotic policy which will surely result in better patient care, safety and healthcare outcomes.

\section{CONCLUSION}

To establish the most suitable empirical treatment for each patient, it is very important to know the microbial epidemiology of each institution. The information obtained from this study allows a better understanding of the microbial etiology of SSIs in local hospitals which may have epidemiological and therapeutic implications. Using the results of this study, an initiative for establishing improved hospital antimicrobial policy and antimicrobial prescribing guidelines can be undertaken. Also the inappropriate and prolonged use of antibiotics should be avoided as this can lead to the development of resistant micro organisms which are even more difficult to get rid of.

The findings in this study has also presented the identification of the pathogens and the categories of operations that must be the target of appropriate prophylaxis at the time of operation or in the very early postoperative period.

\section{ACKNOWLEDGEMENTS}

We would like to thank all the Incharge of participating 
Hospitals, Dean \& Head of Department for their always available guidance.

\section{Compliance With Ethical Standards.}

\section{Conflict Of Interest - None. \\ Funding-None.}

Informed Consent Obtained.

\section{REFERENCES}

1. Khan A.Q, Gul Ar Navi Khan, Firoz M D (2015) Incidence of PostOperative Wound Infections and Bacterial Antibiotic Sensitivity Patterns in Compound Fractures. Natl. J. Integr. Res. Med 6: 21-26.

2. Kos M, Dziewa A, Ksykiewicz-Dorota A, Drop B, Kos M (2016) Nursing care quality and post-operative wound infections. Pol. J. Public Health 126: 13-18.

3. Alebachew T, Yismaw G, Derabe A, Sisay Z (2012) Staphylococcus Aureus Burn Wound Infection Among Patients Attending Yekatit 12 Hospital Burn Unit, Addis Ababa, Ethiopia. Ethiop J Health Sci 22: 209-213.

4. Cruse PJ (1975) Incidence of Wound Infection on the Surgical Services. Surg Clin North Am 55: 1269 - 1275.

5. Centers for Disease control and Prevention: Surgical site infection. Available from: http://www.cdc.gov/HAI/ssi/ssih [Last accessed on 2020 March 19]

6. Malik S, Gupta A, Singh PK, Agarwal J, Singh M. Antibiogram of aerobic bacterial isolates from post- operative wound infections at a tertiary care hospital in india. Journal of Infectious Diseases Antimicrobial Agents. 2011;28:45-51.

7. Satyanarayana V, Prashanth HV, Basavaraj B, Kavyashree AN. Study of surgical site infections in abdominal surgeries. J Clin Diagn Res. 2011;5:935-39.

8. Ahmed MI. Prevalence of nosocomial wound infection among postoperative patients and antibiotics patterns at teaching hospital in Sudan. N Am J Med Sci. 2012;4(1):29-34.

9. Mulu W, Kibru G, Beyene G, Datie M. Postoperative nosocomial infections and antimicrobial resistance patterns of bacterial isolates among patients admitted at FelegeHiwot Referral Hospital, Bahirdar, Ethiopia. EthiopJ Health Sci. 2012;22(1):7-18.

10. Khan AKA, Rashed MR, Banu G. A Study on the Usage Pattern of antimicrobial agents for the prevention of surgical site infections (ssis) in a tertiary care teaching hospital. J Clin Diagn Res. 2013;7(4):671-74.

11. Chakarborty SP, Mahapatra SK, Bal M, Roy S. Isolation and identification of vancomycin resistant Staphylococcus aureus from postoperative pus sample. Al Ameen J Med Sci. 201 1;4(2):152-68.

12. Isibor OJ, Oseni A, Eyaufe A. Incidence of aerobic bacteria and Candida albicans in postoperative wound infections. Afr J microbial Res. 2008:2:288-91.

13. Anguzu JR, Olila D. Drug sensitivity patterns of bacterial isolates from septic post-operative wounds in a regional referral hospital in Uganda. Afr Health Sci. 2007;7(3):148-54

14. Aggarwal A, Khanna S, Arora U, Devi P Correlation of beta-lactamase production/methicillin resistance and phage pattern of Staphylococcus aureus. Indian J Med Sci. 2001;55(5):253-56.

15. Kownhar H, Shankar EM, Vignesh R, Sekar R, Velu V, Rao UA. High isolation rate of Staphylococcus aureus from surgical site infections in an Indian hospital. J AntimicrobChemother. 2008;61:758-60.

16. Eagye KJ, Kim A, Laohavaleeson S, Kuti JL, Nicolau DP. Surgical site infections: does inadequate antibiotic therapy affect patient outcomes? SurgInfect (Larchmt) 2009;10(4):323-31

17. Kaye KS, Anderson DI, Sloane R, Chen LF, Choi Y, Link K, et al. The effect of surgical site infection on older operative patients. J Am Geriatr Soc. 2009:57(1):46-54

18. Masaadeh HA, Jaran AS. Incident of Pseudomonas aeruginosa in postoperative wound infection. Am J Infect Dis. 2009;5:1-6.

19. Fletcher N, Sofianos D, Berkes MB, Obremskey WT. Prevention of perioperative infection. J Bone Joint Surg Am. 2007;89(7):1605-18. 